\title{
Ell3 stimulates 5-FU resistance in a breast cancer cell line
}

\author{
ISAAC KIM ${ }^{1}$, KWANG-SOO KIM ${ }^{1}$, OK-SEON KWON ${ }^{2}$, HYUK-JIN CHA $^{2}$ and KYUNG-SOON PARK ${ }^{1}$ \\ ${ }^{1}$ Department of Biomedical Science, College of Life Science, CHA University, Seongnam-si, Gyeonggi-do 13496; \\ ${ }^{2}$ Department of Life Science, College of Natural Science, Sogang University, Seoul 04107, Republic of Korea
}

Received June 16, 2015; Accepted October 24, 2016

DOI: $10.3892 / \mathrm{ol} .2017 .5996$

\begin{abstract}
Ell3 is an RNA polymerase II transcription elongation factor that acts as a negative regulator of p53 expression, and regulates cell proliferation and survival. Recent studies by our group have demonstrated that ectopic expression of El13 in breast cancer cell lines enhances cell proliferation, potentiates cancer stem cell properties, and promotes 5-Fluorouracil (5-FU) resistance. In the present study, the underlying mechanism for the induction of 5-FU resistance was investigated in Ell3 over-expressing MCF-7 cells (E113 OE cells). By comparing the gene expression profiles of Ell3 OE cells with control cells, the present data revealed that Lipocalin2 (LCN2) and Wnt signaling activity are associated with 5-FU resistance of Ell3 OE. siRNA-mediated suppression of LCN2 reversed 5-FU resistance in Ell3 OE cells. Chemical inhibition of Wnt signaling also reversed 5-FU resistance in Ell3 OE cells. Furthermore, the expression levels of survivin, which is a direct transcriptional target of $\mathrm{Wnt} / \beta$-catenin and an inhibitor of apoptosis, were markedly elevated when Ell3 OE cells were treated with 5-FU, as detected by western blot analysis. These findings suggest that enhanced expression of LCN2 and activation of the Wnt signaling pathway may induce 5-FU resistance in Ell3 OE cells as a means of evading apoptosis.
\end{abstract}

\section{Introduction}

Breast cancer is a common malignancy in women and is a significant cause of mortality. In spite of treatment, $>4,000$ individuals succumbed to breast cancer in the US in 2016 (1).

The testis-specific RNA polymerase II elongation factor (Ell3) is known to increase the oncogenicity of breast cancer cell lines by regulating the expression of cell cycle regulators through the ERK signaling pathway and via the induction of drug resistance through unknown mechanisms (2). The

Correspondence to: Professor Kyung-Soon Park, Department of Biomedical Science, College of Life Science, CHA University, 335 Pangyo-ro, Bundang-gu, Seongnam-si, Gyeonggi-do 13496, Republic of Korea

E-mail:kspark@cha.ac.kr

Key words: Ell3, 5-Fluorouracil resistance, breast cancer, Lipocalin2, WNT signaling
C-terminal domain of Ell3 exhibits strong similarities to that of the eleven-nineteen lysine-rich leukemia gene, which acts as a negative regulator of p53 and regulates cell proliferation and survival (3-5). Furthermore, Ell3 occupies enhancers in embryonic stem cells and Ell3 binding to inactive or poised enhancers is essential for stem cell specification (6).

Lipocalin-2 (LCN2), also known as neutrophil gelatinase-associated lipocalin, is a member of the lipocalin protein family and is upregulated in various types of epithelial cancer, including breast, lung, thyroid, esophageal and pancreatic duct adenocarcinomas (7-9). LCN2 has been reported to promote drug resistance and tumor growth, and enhance tumor cell invasion through its physical association with matrix metalloproteinase-9 (10). The functions of LCN2 during cancer progression are yet to be fully elucidated. In human breast cancer, LCN2 expression has been associated with markers of poor prognosis, including estrogen receptor (ER)-negative status, poor histological grading and lymph node metastasis, and LCN2 been shown to be an independent prognostic marker for decreased survival $(11,12)$. LCN2 has been demonstrated to suppress apoptosis in thyroid, lung, breast and pancreatic duct adenocarcinomas $(7,13)$.

The Wnt signaling pathway, named after its most upstream ligands, the Wnts, is involved in various differentiation events during embryonic development. Wnt signaling is also associated with tumor formation (14). In the canonical Wnt signaling cascade, adenomatous polyposis coli, axin and glycogen synthase kinase (GSK) constitute the so-called 'destruction complex', which controls the stability of $\beta$-catenin (15). In cells that receive the Wnt signal, GSK is presumed not to phosphorylate $\beta$-catenin. As a consequence, $\beta$-catenin accumulates and forms nuclear complexes with transcription factors (15). In breast cancer, Wnt stimulates tumor cell motility; conversely, Wnt pathway blockade reduces motility (16). Furthermore, Wnt signaling has been demonstrated to promote stem cell activity in mammosphere assays of mammary gland cells (17).

In lung cancer, Wnt signaling has an essential role in maintaining highly resistant cancer stem cells, and in regulating cell cycle, metastasis and apoptosis. Thus, Wnt antagonists decrease metastasis and induce apoptosis (18). Wnt signaling has also been reported to be associated with drug resistance $(19,20)$. Dysregulation of the $\mathrm{Wnt} / \beta$-catenin pathway was shown to be involved in pancreatic cancer chemoresistance (19). Drug resistance of colon cancer cells to 5-fluorouracil (5-FU) and irinotecan treatment has been linked to Wnt signaling in a previous study (20). These features of Wnt signaling are 
associated with apoptosis $(21,22)$. Wnt signaling regulates the early and late stages of apoptosis during development and upon cellular injury of neurons, endothelial cells, vascular smooth muscle cells and cardiomyocytes (20). In human melanoma, inhibition of Wnt-2 signaling, by either a novel monoclonal antibody against human Wnt-2 ligand or Wnt-2 small interfering RNA (siRNA), downregulated $\beta$-catenin and survivin, and induced apoptosis (22).

Survivin, which is a member of the family of apoptosis protein inhibitors, functions as a key regulator of mitosis and programmed cell death (23). Survivin has been identified as a direct transcriptional target of Wnt/ $\beta$-catenin (24), which involves the recognition of discrete T-cell factor-4-binding elements in the survivin promoter. Functionally, forced expression of non-destructible $\beta$-catenin readily increases survivin levels and supports survivin-mediated cytoprotection (25).

Resistance to chemotherapy is a major problem facing current cancer treatment. The mechanisms of resistance to cytotoxic chemotherapeutics share various features, including alterations in the drug target, activation of pro-survival pathways and ineffective induction of cell death (26).

Our group have previously demonstrated that ectopic expression of Ell3 in breast cancer cell lines induced 5-FU drug resistance (2). To understand the underlying mechanism resistance of Ell3 over-expressing MCF-7 breast cancer cell line (El13 OE) to 5-FU, the present study compared gene expression profiles of Ell3 OE cells with wild type (control) cells after treatment with 5-FU. A number of genes and signals related to drug resistance were activated in Ell3 OE cells by treatment with 5-FU. The possible role of Ell3 as an upstream regulator of these genes and signaling pathways is discussed herein.

\section{Materials and methods}

Cell culture and reagents. MCF-7 cell lines were purchased from American Type Culture Collection (Manassas, VA, USA). MCF-7 cells were cultured in Dulbecco's modfiied Eagle medium supplemented with $10 \%$ fetal bovine serum and $1 \%$ penicillin/streptomycin (all Invitrogen; Thermo Fisher Scientific, Inc., Waltham, MA, USA). El13 OE, which are Ell3-overexpressing MCF-7 cell lines, were generated by chromosomal integration of an Ell3 expression plasmid, which was constructed by cloning PCR-amplified Ell3 cDNA into a modified pcDNA3.1 vector (Invitrogen; Thermo Fisher Scientific, Inc.) in which the CMV promoter was replaced by an EF1a promoter. Three independent MCF-7 cell lines were established for Ell3-OE and MCF-7, respectively, and all experiments were repeated in each cell line to confirm the results. Nonspecific control siRNAs and siRNAs targeting Ell3 were purchased from Dharmacon (Lafayette, CO, USA). MCF-7 cells were transfected with either siRNA or plasmids using Lipofectamine 2000 (Invitrogen; Thermo Fisher Scientific, Inc.) according to the manufacturer's instructions.

Microarray analysis. Biotinylated cRNAs were prepared from 500 ng total RNA according to the standard Affymetrix protocol (Affymetrix, Santa Clara, CA, USA). Following fragmentation, $12 \mu \mathrm{g}$ aRNA was hybridized for $16 \mathrm{~h}$ at $45^{\circ} \mathrm{C}$ using a GeneChip Human Genome Array. GeneChips were washed and stained in the Affymetrix Fluidics Station 450 then were scanned using the Affymetrix GeneChip Scanner 3000 7G. Data were analyzed via Robust Multi-array Analysis using Affymetrix default analysis settings and global scaling as the normalization method. The trimmed mean target intensity of each array was arbitrarily set to 100 . Normalized and log-transformed intensity values were subsequently analyzed using GeneSpring GX 12.6 (Agilent Technologies, Santa Clara, CA, USA). Fold-change filters included the requirement that the genes be expressed at levels at least $150 \%$ of control levels for upregulated genes, and $<66 \%$ of control levels for downregulated genes. Hierarchical clustering data were clustered groups that behaved similarly across experiments using GeneSpring GX 12.6 (Agilent Technologies). The clustering algorithm used was Euclidean distance at average linkage.

Reverse transcription-quantitative polymerase chain reaction $(R T-q P C R)$. Total RNA was isolated from the MCF-7 cell line using TRIzol reagent (Invitrogen; Thermo Fisher Scientific, Inc.) and $2 \mu \mathrm{g}$ total RNA was reverse-transcribed into cDNA using the SuperScript II First-Strand Synthesis System (Invitrogen; Thermo Fisher Scientific, Inc.), which contained Primer, Script reverse transcriptase, RNase inhibitor, deoxynucleotide mixture and reaction buffer, according to the manufacturer's instructions. qPCR was performed in triplicate using a Quantitect SYBR Green PCR kit (Qiagen, Germantown, MD, USA) and CFX96 Real-time System (Bio-Rad Laboratories, Inc., Hercules, CA, USA) under the following cycling conditions: $95^{\circ} \mathrm{C}$ for $30 \mathrm{sec}$ for $35 \mathrm{cycles}, 55^{\circ} \mathrm{C}$ for $30 \mathrm{sec}$ for 40 cycles and $70^{\circ} \mathrm{C}$ for $30 \mathrm{sec}$ for 30 cycles, respectively. Qiagen $2 X$ SYBR mix $(10 \mu \mathrm{l})$, primers (10 pmol; forward and reverse each $1 \mu \mathrm{l})$, cDNA $(1 \mu \mathrm{g})$ and deionized water $(20 \mu \mathrm{l})$ were used. For quantification, target gene expression was normalized to the expression of glyceraldehyde 3-phosphate dehydrogenase (GAPDH). The following primer sequences were used: GAPDH forward, 5'-GGGTGTGAACCATGAGAA-3' and reverse, 5'-GTCTTC TGGGTGGCAGTGAT-3'; and LCN2 forward, 5'-CCTGGA GACATTGGGGACTTCA-3' and reverse, 5'-GCCACTGCC TTCATAGTCAAACAC-3'. The data was normalized using the $2^{-\triangle \Delta \mathrm{Cq}}$ method (27).

Immunoblot assay. For protein analysis, cells were washed twice with cold phosphate-buffered saline (PBS) and lysed with tissue lysis buffer (20 mM Tris-base, $\mathrm{pH} 7.4,137 \mathrm{mM} \mathrm{NaCl}$, 2 mM EDTA, 1\% Triton X-100, 25 mM $\beta$-glycerophosphate, $2 \mathrm{mM}$ sodium pyrophosphate, $10 \%$ glycerol, $1 \mathrm{mM}$ sodium orthovanadate, $1 \mathrm{mM}$ phenylmethylsulfonyl fluoride and $1 \mathrm{mM}$ benzamidine). Lysates were centrifuged at 16,600 x $g$ at $4{ }^{\circ} \mathrm{C}$ for $10 \mathrm{~min}$ to remove cellular debris. Whole-cell extracts were prepared and $50 \mathrm{mg}$ of protein was separated by $10 \%$ SDS-PAGE and transferred to polyvinylidene difluoride membranes (Bio-Rad Laboratories, Inc.). The membranes were blocked with blocking solution (5\% skim milk in TBST; $50 \mathrm{mM}$ Tris-base, $\mathrm{pH} 7.4,0.15 \mathrm{M} \mathrm{NaCl}$ and $0.1 \%$ Tween-20) for $1 \mathrm{~h}$ prior to subsequent incubation with anti-survivin (catalog no. sc-17779; 1:1,000 dilution; Santa Cruz Biotechnology, Inc., Dallas, TX, USA) and anti- $\beta$-actin (catalog no. sc-47778; 1:1,000 dilution; Santa Cruz Biotechnology, Inc.) primary antibodies overnight at $4^{\circ} \mathrm{C}$. Following this, the membranes were washed three times for $10 \mathrm{~min}$ in TBST and incubated with an anti-mouse IgG secondary antibody (catalog no. sc-516102; 
A

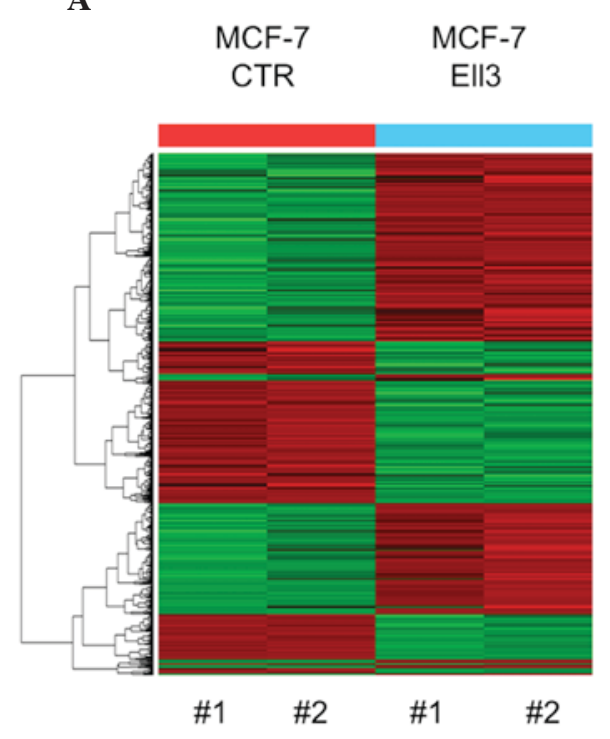

B

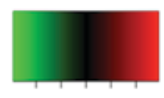

$\begin{array}{lll}-1 & 0 & 1\end{array}$

Z-score

\section{$\%$ of total significant changes in expression}

Aging

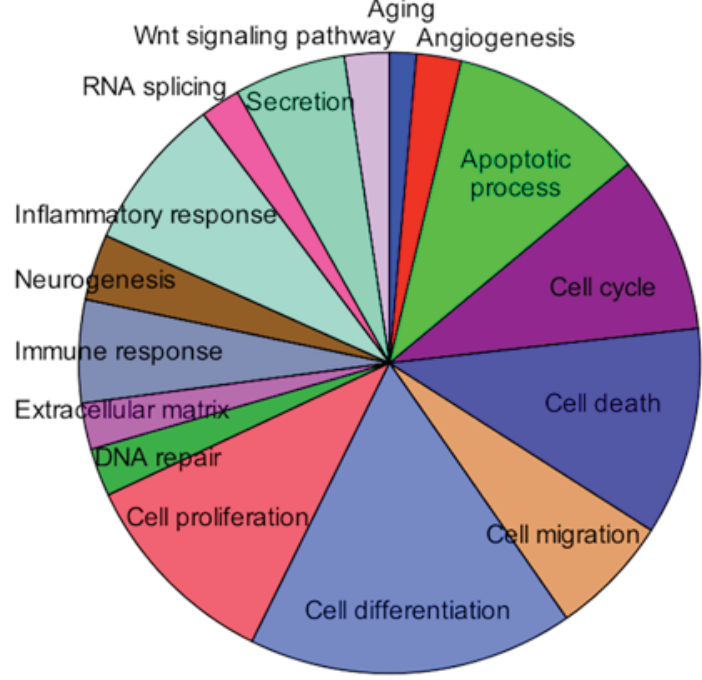

C

\begin{tabular}{|l|l|l|l|l|l|}
\hline & Target ID & Symbol & Definition & Accession & Fold Ell3OE/CTR \\
\hline \#1 & \multirow{2}{*}{ 11757634_a_at } & LCN2 & Lipocalin-2 & NM-005564 & 22.99973 \\
\cline { 1 - 1 } \cline { 5 - 6 } & & & & & 24.877024 \\
\hline
\end{tabular}

D

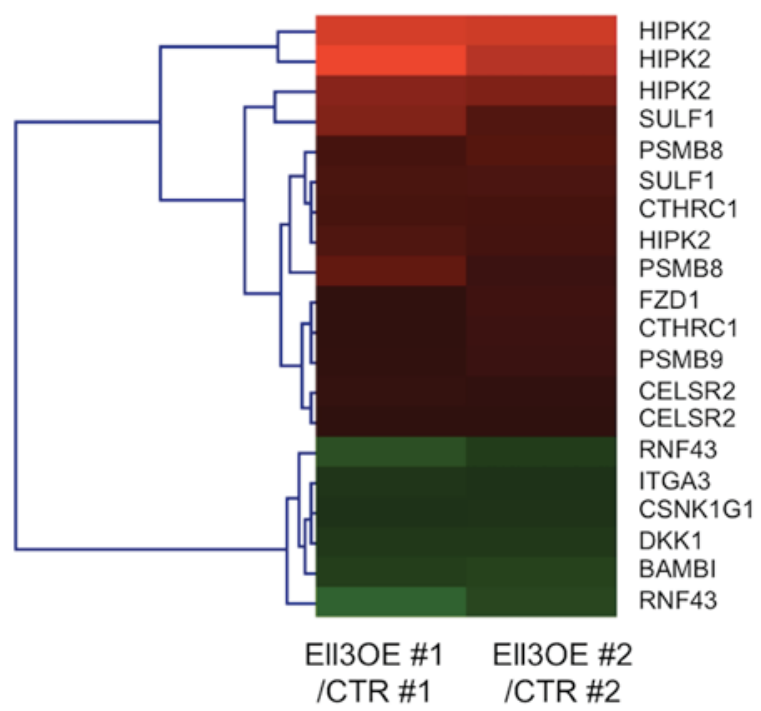

$\begin{array}{lll}-3 & 0 & 3\end{array}$

EII3OE/CTR

Fold ratio

Figure 1. Microarray analysis of the effect of 5-FU on gene expression in El13 OE and control cells. (A) Genome-wide expression analysis of control and El13 OE cells. Experiments were repeated twice. Significant changes (>2-fold) are indicated by color (red, upregulated; green, downregulated). (B) Diagram of significant changes in gene expressions (>2-fold in El13 OE cells relative to control cells) according to functional category. (C) Microarray analysis of LCN2 expression in El13 OE compared with control cells. Experiments were repeated twice. (D) Heat map of Wnt signaling-related genes that were altered in Ell3 OE by >2-fold compared with control cells. CTR, control MCF-7 cells; El13 OE, Ell3 overexpressing MCF-7 cells; 5-FU, 5-Fluorouracil.

1:5,000 dilution; Santa Cruz Biotechnology, Inc.) for $1 \mathrm{~h}$ at $37^{\circ} \mathrm{C}$. Protein quantification was performed with ELISA DuoSet ${ }^{\circledR}$ Human Carbonic Anhydrase IX (R\&D Systems, Inc., Minneapolis, MN, USA). The signals were analyzed following treatment with TMB substrate and visualized by the ChemiDoc $^{\mathrm{TM}}$ Touch Imaging system (Bio-Rad Laboratories, Inc.).
Water-soluble tetrazolium salt-1 cell proliferation assay. MCF-7 cells $\left(4 \times 10^{4}\right.$ cells per well) were seeded into a 24 -well cell culture plate. Epigallocatechin-3-gallate (EGCG; $20 \mu \mathrm{M}$ ) or IWP-2 $(5 \mu \mathrm{M})$, which are LCN2 and Wnt signaling pathway inhibitors, respectively, were added to the cultures at appropriate concentrations for $48 \mathrm{~h}$. Cell proliferation assays were performed using an EZ-CyTox Enhanced Cell Viability Assay 
A

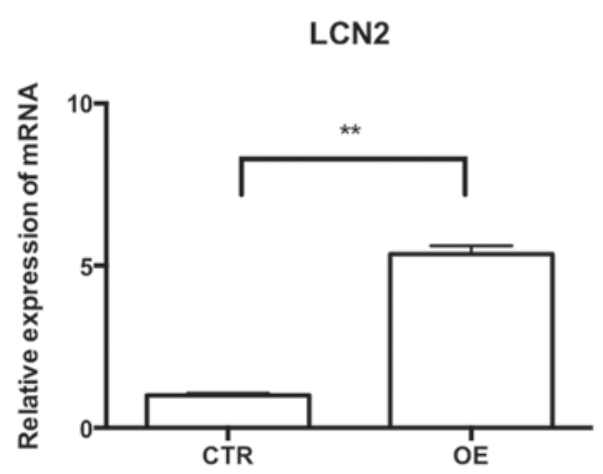

B

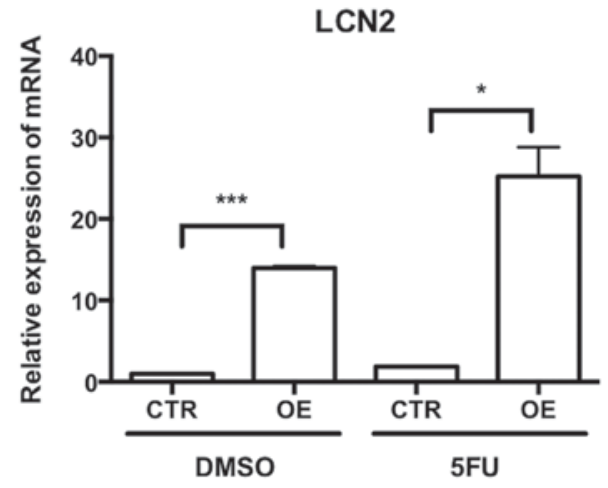

C

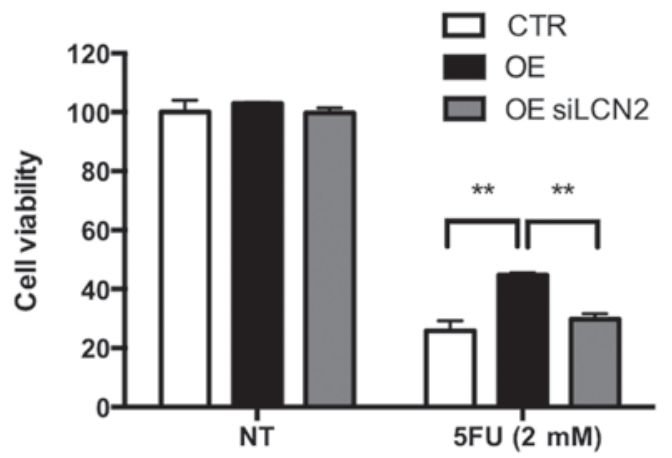

Figure 2. Effect of LCN2 expression on gene expression and viability in El13 OE cells. (A) Relative LCN2 expression in control and El13 OE cells was analyzed by RT-qPCR. (B) Alterations in LCN2 expression levels in control and Ell3 OE cells after 5-FU treatment were analyzed by real-time RT-qPCR. (C) Cell viabilities of control, Ell3 OE, and El13 OE treated with siLCN2 were analyzed by water-soluble tetrazolium salt 1 assay. Cell viability was measured under mock treatment and $2 \mathrm{mM} 5-\mathrm{FU}$ treatment conditions. ${ }^{*} \mathrm{P}<0.05 ;{ }^{* *} \mathrm{P}<0.01 ;{ }^{* * *} \mathrm{P}<0.005$. CTR, control MCF-7 cells; OE, Ell3 over-expressing MCF-7 cells; siLCN2, siRNA targeting LCN2; DMSO, dimethyl sulfoxide; NT, nonspecific treatment; 5-FU, 5-Fluorouracil; RT-qPCR, reverse transcription-quantitative polymerase chain reaction.

kit (Daeil Lab Services Co., Seoul, Korea). Absorbance at $450 \mathrm{~nm}$ was measured using a microplate reader.

Immunocytochemical staining. MCF-7 cells were cultured on cover slips. Following washing twice with PBS, cells were fixed with $4 \%$ paraformaldehyde for $15 \mathrm{~min}$. Cover slips were washed three times with PBS and the cells were permeabilized with $0.1 \%$ Tween-20 in PBS for 20 min followed by blocking for 30 min using blocking buffer (5\% bovine serum albumin in PBS). Following overnight incubation with the primary antibodies, the cover slips were washed three times with PBS and treated with Alexa Flour 594 donkey anti-rabbit IgG (A21207; Invitrogen; Thermo Fisher Scientific, Inc.) for $1 \mathrm{~h}$ in the dark. Cover slips were then washed three times in PBS and mounted with VECTASHIELD Mounting Medium with DAPI (H-1200; Vector Laboratories, Burlingame, CA, USA). Images were captured using a Leica confocal laser scanning microscopy system (Leica Microsystems GmbH, Wetzlar, Germany).

Statistical analysis. Graphical data are presented as the mean \pm standard deviation. Each experiment was performed at least three times and subjected to statistical analysis. Statistical significance between two groups was determined using the Student's t-test. $\mathrm{P}<0.05$ was considered to indicate a statistically significant difference. All statistical analyses were performed using GraphPad Prism 6.0 software (GraphPad Software, Inc., La Jolla, CA, USA).

\section{Results}

5-FU treatment of Ell3 OE cells induces upregulation of LCN2 gene expression and Wnt signaling. cDNA microarray analysis was performed to compare the gene expression profiles of Ell3 $\mathrm{OE}$ and control MCF-7 cells after 5-FU treatment. Fig. 1A indicates the extensive alterations of total gene expression that were detected in the present study. Among 20,811 genes in El13 OE cells treated with 5-FU, expression levels of 694 genes $(\sim 3.33 \%)$ were significantly altered by $>2$-fold (450 genes were upregulated and 244 genes were downregulated). Genes with altered expression were classified according to functional categories (Fig. 1B). The cell differentiation category had the most genes with altered expression $(0.65 \%)$, followed by the cell proliferation category $(0.41 \%)$. The cell death and apoptotic process categories represented 0.36 and $0.35 \%$, respectively. It was noted that expression of LCN2, which is able to alter the sensitivity of certain types of cancers to chemotherapeutic drugs (28), was upregulated by $>20$-fold in El13 OE compared with control cells (Fig. 1C). The Kyoto Encyclopedia of Genes and Genomes (KEGG) is a knowledge base for systematic analysis of gene functions in terms of the networks of genes and molecules. The KEGG pathway database consists of graphical 
A

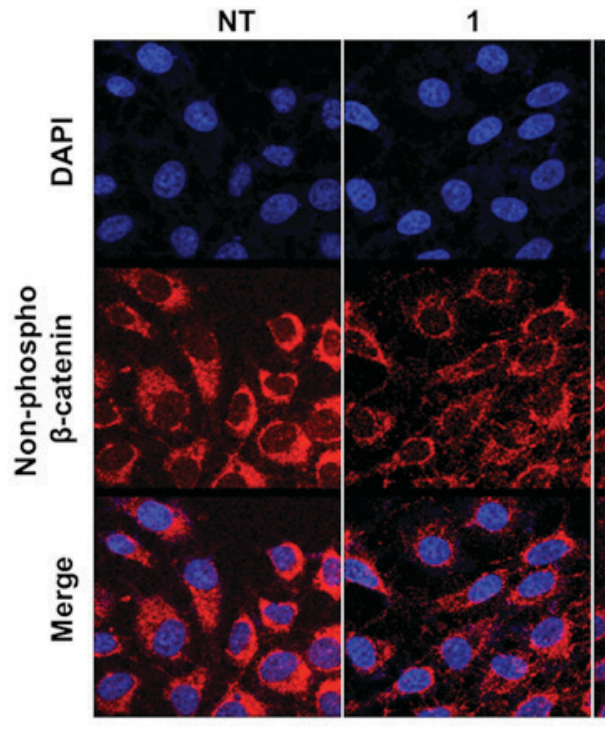

2

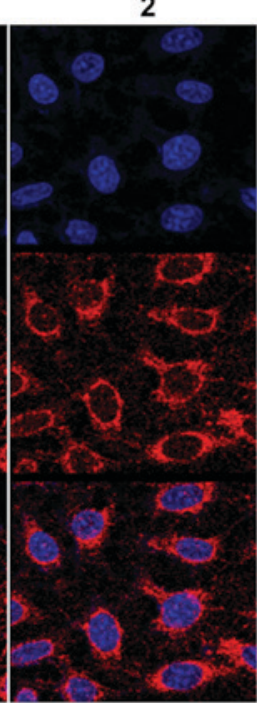

4

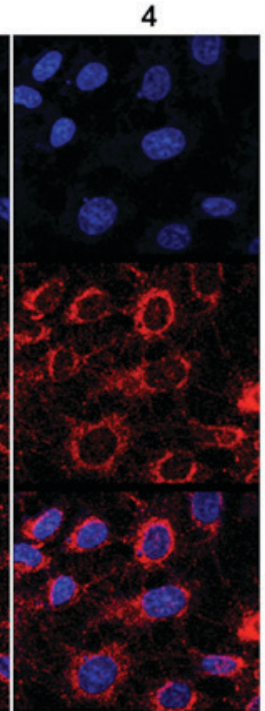

[5FU] (mM)

B

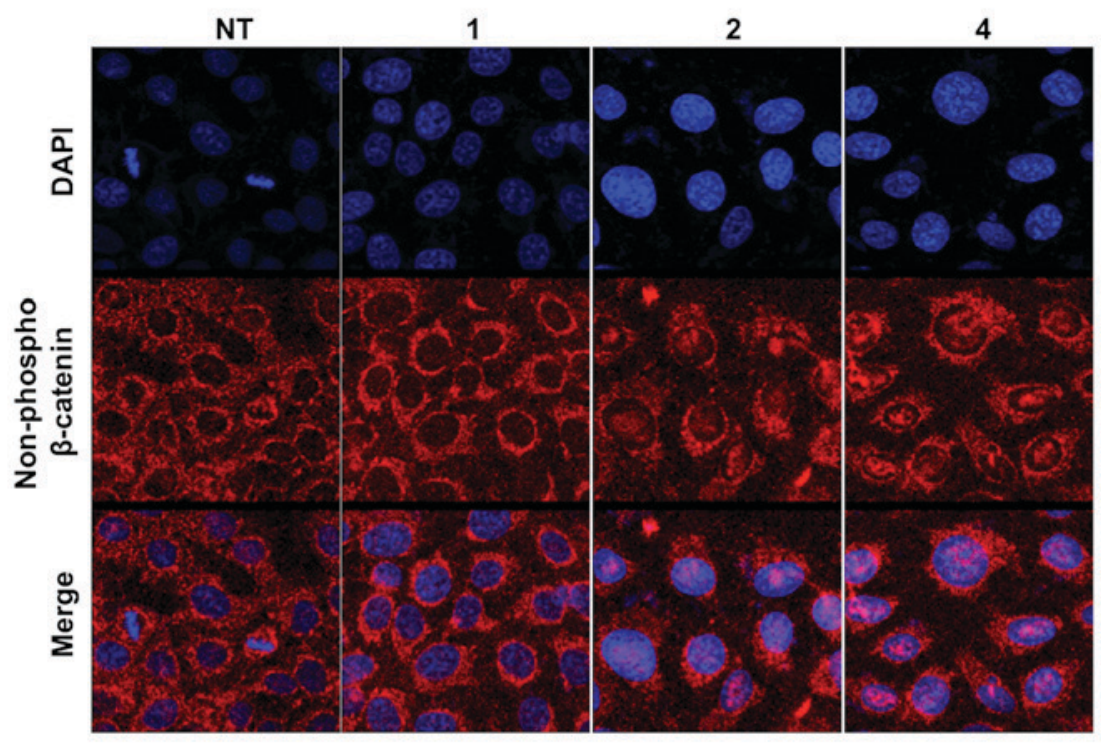

$[5 F U](\mathrm{mM})$

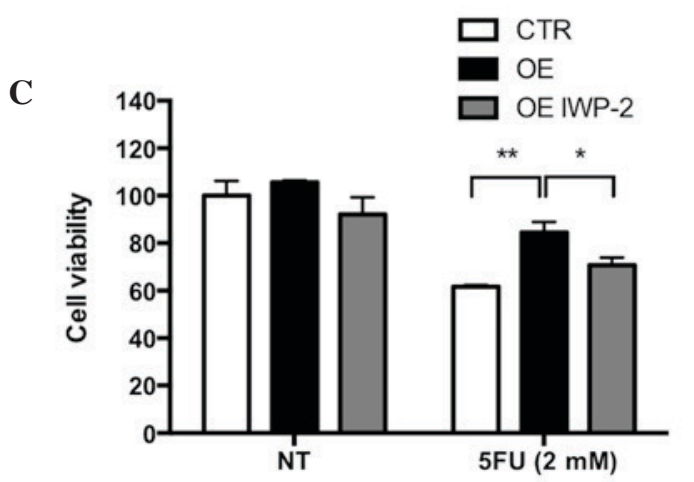

D

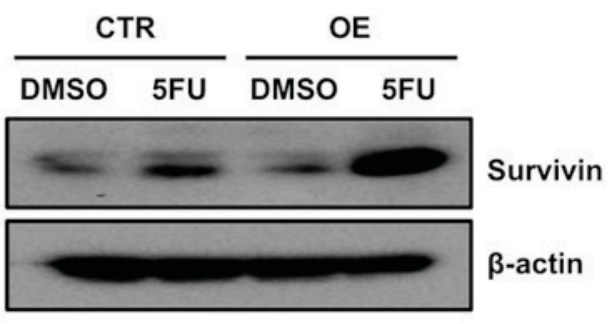

Figure 3. Effect of enhanced WNT signaling in El13 OE cells. Localization of non-phosphorylated $\beta$-catenin was analyzed by immunocytochemical staining in (A) control MCF-7 and (B) El13 OE treated with various 5-FU concentrations. (C) Cell viabilities of control, Ell3 OE and Ell3 OE cells treated with IWP were analyzed by water-soluble tetrazolium salt 1 assay. Cell viability was measured under mock treatment and $2 \mathrm{mM} \mathrm{5-FU} \mathrm{treatment} \mathrm{conditions.} \mathrm{(D)} \mathrm{Expression}$ levels of survivin in control and El13 OE cells under mock-treated and $2 \mathrm{mM} 5$-FU treatment were analyzed by immunoblot assay. ${ }^{*} \mathrm{P}<0.05$; ${ }^{* *} \mathrm{P}<0.01$. CTR, control MCF-7 cells; OE, El13 over-expressing MCF-7 cells; NT, nonspecific treatment; IWP-2, inhibitor of WNT processing; 5-FU, 5-Fluorouracil.

diagrams of biochemical pathways including the majority of the known metabolic pathways and some of the known regulatory pathways (29). The KEGG pathway program was utilized for the genes with >2-fold altered expression on the microarray analysis. Notably, $66.6 \%$ of the Wnt signaling pathway-related genes were activated in Ell3 OE cells (Fig. 1D) and several genes known to inhibit Wnt signaling were downregulated. These results suggested that activation ofLCN2 and Wnt 
signaling pathway genes was the primary cause of 5-FU drug resistance in Ell3 OE cells.

LCN2 reinforces 5-FU drug resistance in Ell3 OE cells. To demonstrate the role of LCN2 and Wnt signaling in the resistance of El13 OE cells to 5-FU, the activation of LCN2 expression in Ell3 OE cells was confirmed by RT-qPCR analysis. Notably, LCN2 expression was activated in Ell3 OE cells in the absence of 5-FU and the expression level was further increased after 5-FU treatment (Fig. 2A and B). These results suggested that overexpression of LCN2 may be the cause of 5-FU resistance in Ell3 OE cells. To confirm this possibility, whether suppression of LCN2 expression in Ell3 OE cells diminished 5-FU resistance. siLCN2-transfected Ell3 OE cells were subsequently supplemented with 5 -FU; cell viability was similar to the wild type, which indicated that overexpression of LCN2 was the main cause of 5-FU resistance (Fig. 2C). Prior to 5-FU treatment, El13 OE cells were pretreated with EGCG, which is a chemical inhibitor of LCN2 activity, and drug resistance was significantly decreased (data not shown). These results suggested that LCN2 expression induced 5-FU drug resistance in Ell3 OE cells.

Wnt signaling is associated with 5-FU drug resistance in Ell3 $O E$ cells. Activation of the Wnt signaling pathway induced accumulation of non-phosphorylated $\beta$-catenin in the nucleus of a cell (14). To confirm that the Wnt signaling pathway was activated in Ell3 OE cells upon 5-FU treatment, nuclear localization of non-phosphorylated $\beta$-catenin was analyzed via immunocytochemical (ICC) staining. As shown in Fig. 3A and $\mathrm{B}$, non-phosphorylated $\beta$-catenin accumulated in the nucleus of Ell3 OE after treatment with 2 and $4 \mathrm{mM} 5-\mathrm{FU}$, whereas $\beta$-catenin in control cells was detected in the cytosol under the same conditions. To further elucidate the role of Wnt signaling in the resistance of Ell3 OE cells to 5-FU, the effect of IWP-2, which is an inhibitor of Wnt signaling, on the resistance of Ell3 OE cells to 5-FU was investigated. As hypothesized, cell viability of Ell3 OE after 5-FU treatment was significantly decreased in the presence of IWP-2 (Fig. 3C).

Activation of Wnt signaling was associated with enhanced expression of survivin, a member of the inhibitor of apoptosis protein family (24). Therefore, whether survivin expression was increased in Ell3 OE cells was examined in the presence or absence of 5-FU. As shown in Fig. 3D, survivin was markedly increased in Ell3 OE cells treated with 5-FU, but not in control cells. These results indicated that Wnt signaling was activated in Ell3 OE cells after 5-FU treatment and supported the possibility that drug resistance to 5-FU was mediated via the inhibition of apoptosis-related protein activity.

\section{Discussion}

The present study investigated the drug resistant mechanism of Ell3 OE upon 5-FU treatment. The findings showed that LCN2 expression in Ell3 OE was higher than control and LCN2 expression was increased after 5-FU treatment in the Ell3 OE groups, as compared with the control. Elevated cell viability in Ell3 OE was decreased by treatment with siLCN2 and the LCN2 chemical inhibitor, EGCG. Expression of LCN2 has been reported to be associated with the anticancer drug resistance of several cancers, including renal cell carcinoma and pancreatic duct adenocarcinomas $(30,31)$, which implies the pivotal role of LCN2 in the drug resistance of cancer cells. Therefore, investigation of the role of Ell3 in the expression of LCN2 may provide important insight into the regulatory mechanism of LCN2 expression.

The present findings also showed that Wnt signaling and survivin expression were enhanced in El13 OE cells and that inhibition of Wnt signaling resulted in the suppression of 5-FU resistance in Ell3 OE cells. In contrast to LCN2 expression, Wnt signaling and survivin expression were only increased after 5-FU treatment. This result suggested that LCN2 expression, which was activated in Ell3 OE cells in the absence of 5-FU, was not associated with Wnt signaling. Furthermore, the resistance of Ell3 OE cells to 5-FU was induced independently by LCN2 activity and Wnt signaling. Since Wnt signaling has a crucial role in tumor development and drug resistance, understanding the role of Wnt signaling will aid the development of effective strategies to overcome chemotherapeutic resistance in various types of cancer.

\section{Acknowledgements}

The present study was supported by the Ministry of Education, Science, and Technology of the Korean government (grant nos. 2012M3A9C6050367 and 2015R1A2A2A01003498).

\section{References}

1. Siegel RL, Miller KD and Jemal A: Cancer statistics, 2016. CA Cancer J Clin 66: 7-30, 2016.

2. Ahn HJ, Kim G and Park KS: Ell3 stimulates proliferation, drug resistance, and cancer stem cell properties of breast cancer cells via a MEK/ERK-dependent signaling pathway. Biochem Biophys Res Commun 437: 557-564, 2013.

3. Miller T, Williams K, Johnstone RW and Shilatifard A: Identification, cloning, expression, and biochemical characterization of the testis-specific RNA polymerase II elongation factor ELL3. J Biol Chem 275: 32052-32056, 2000.

4. Maki K, Mitani K, Yamagata T, Kurokawa M, Kanda Y, Yazaki Y and Hirai H: Transcriptional inhibition of p53 by the MLL/ MEN chimeric protein found in myeloid leukemia. Blood 93: 3216-3224, 1999.

5. Johnstone RW, Gerber MA, Landewe T, Tollefson A, Wold WS and Shilatifard A: Functional analysis of the leukemia protein ELL: Evidence for a role in the regulation of cell growth and survival. Mol Cell Biol 21: 1672-1681, 2001.

6. Lin C, Garruss AS, Luo Z, Guo F and Shilatifard A: The RNA Pol II elongation factor Ell3 marks enhancers in ES cells and primes future gene activation. Cell 152: 144-156, 2013.

7. Iannetti A, Pacifico F, Acquaviva R, Lavorgna A, Crescenzi E, Vascotto C, Tell G, Salzano AM, Scaloni A, Vuttariello E, et al: The neutrophil gelatinase-associated lipocalin (NGAL), a NF-kappaB-regulated gene, is a survival factor for thyroid neoplastic cells. Proc Natl Acad Sci USA 105: 14058-14063, 2008.

8. Zhang H, Xu L, Xiao D, Xie J, Zeng H, Wang Z, Zhang X, Niu Y, Shen Z, Shen J, et al: Upregulation of neutrophil gelatinase-associated lipocalin in oesophageal squamous cell carcinoma: Significant correlation with cell differentiation and tumour invasion. J Clin Pathol 60: 555-561, 2007.

9. Leng X, Ding T, Lin H, Wang Y, Hu L, Hu J, Feig B, Zhang W, Pusztai L, Symmans WF, et al: Inhibition of lipocalin 2 impairs breast tumorigenesis and metastasis. Cancer Res 69: 8579-8584, 2009.

10. Yang J, McNeish B, Butterfield C and Moses MA: Lipocalin 2 is a novel regulator of angiogenesis in human breast cancer. FASEB J 27: 45-50, 2013. 
11. Gruvberger S, Ringnér M, Chen Y, Panavally S, Saal LH, Borg A, Fernö M, Peterson C and Meltzer PS: Estrogen receptor status in breast cancer is associated with remarkably distinct gene expression patterns. Cancer Res 61: 5979-5984, 2001.

12. Bauer M, Eickhoff JC, Gould MN, Mundhenke C, Maass N and Friedl A: Neutrophil gelatinase-associated lipocalin (NGAL) is a predictor of poor prognosis in human primary breast cancer. Breast Cancer Res Treat 108: 389-397, 2008.

13. Tong Z, Wu X, Ovcharenko D, Zhu J, Chen CS and Kehrer JP Neutrophil gelatinase-associated lipocalin as a survival factor. Biochem J 391: 441-448, 2005

14. Giles RH, van Es JH and Clevers H: Caught up in a Wnt storm: Wnt signaling in cancer. Biochim Biophys Acta 1653: 1-24, 2003.

15. van Noort M, Meeldijk J, van Der Zee R, Destree O and Clevers H: Wnt signaling controls the phosphorylation status of beta-catenin. J Biol Chem 277: 17901-17905, 2002.

16. Matsuda Y, Schlange T, Oakeley EJ, Boulay A and Hynes NE: WNT signaling enhances breast cancer cell motility and blockade of the WNT pathway by sFRP1 suppresses MDA-MB-231 xenograft growth. Breast Cancer Res 11: R32, 2009.

17. Many AM and Brown AM: Both canonical and non-canonical Wnt signaling independently promote stem cell growth in mammospheres. PLoS One 9: e101800, 2014.

18. Zhang Y, Zhang X, Huang J and Dong Q: Wnt signaling regulation of stem-like properties in human lung adenocarcinoma cell lines. Med Oncol 32: 157, 2015.

19. Cui J, Jiang W, Wang S, Wang L and Xie K: Role of Wnt/ $\beta$-catenin signaling in drug resistance of pancreatic cancer. Curr Pharm Des 18: 2464-2471, 2012

20. Chikazawa N, Tanaka H, Tasaka T, Nakamura M, Tanaka M, Onishi $\mathrm{H}$ and Katano M: Inhibition of Wnt signaling pathway decreases chemotherapy-resistant side-population colon cancer cells. Anticancer Res 30: 2041-2048, 2010.

21. Pećina-Slaus N: Wnt signal transduction pathway and apoptosis: A review. Cancer Cell Int 10: 22, 2010.
22. You L, He B, Xu Z, Uematsu K, Mazieres J, Fujii N, Mikami I, Reguart N, McIntosh JK, Kashani-Sabet M, et al: An anti-Wnt-2 monoclonal antibody induces apoptosis in malignant melanoma cells and inhibits tumor growth. Cancer Res 64: 5385-5389, 2004.

23. Mita AC, Mita MM, Nawrocki ST and Giles FJ: Survivin: Key regulator of mitosis and apoptosis and novel target for cancer therapeutics. Clin Cancer Res 14: 5000-5005, 2008.

24. Fodde R and Brabletz T: Wnt/beta-catenin signaling in cancer stemness and malignant behavior. Curr Opin Cell Biol 19: $150-158,2007$.

25. Kim PJ, Plescia J, Clevers H, Fearon ER and Altieri DC: Survivin and molecular pathogenesis of colorectal cancer. Lancet 362: 205-209, 2003.

26. Holohan C, Van Schaeybroeck S, Longley DB and Johnston PG: Cancer drug resistance: An evolving paradigm. Nat Rev Cancer 13: 714-726, 2013.

27. Livak KJ and Schimittgen TD: Analysis of relative gene expression data using real-time quantitative PDCR and the 2 delta delta method. Methods 25: 402-408, 2001.

28. Chappell WH, Abrams SL, Franklin RA, LaHair MM, Montalto G, Cervello M, Martelli AM, Nicoletti F, Candido S, Libra M, et al: Ectopic NGAL expression can alter sensitivity of breast cancer cells to EGFR, Bcl-2, CaM-K inhibitors and the natural plant product berberine. Cell Cycle 11: 4447-4461, 2012.

29. Ogata H, Goto S, Sato K, Fujibuchi W, Bono H and Kanehisa M: KEGG: Kyoto encyclopedia of genes and genomes. Nucleic Acids Res 27: 29-34, 1999.

30. Yu DS, Wu CL, Ping SY, Huang YL and Shen KH: NGAL can alternately mediate sunitinib resistance in renal cell carcinoma. J Urol 192: 559-566, 2014.

31. Leung L, Radulovich N, Zhu CQ, Organ S, Bandarchi B, Pintilie M, To C, Panchal D and Tsao MS: Lipocalin2 promotes invasion, tumorigenicity and gemcitabine resistance in pancreatic ductal adenocarcinoma. PLoS One 7: e46677, 2012. 